\title{
Optimal Clustering AND ROUTING For WIRELESS SENSOR NETWORK BASED ON CUCKOO SEARCH
}

\author{
Soumitra Das ${ }^{1}$, Barani $\mathrm{S}^{2}$, Sanjeev $\mathrm{Wagh}^{3}$ and S.S. Sonavane ${ }^{4}$ \\ ${ }^{1}$ Research Scholar,Dept. of Computer Science and Engineering, Sathyabama University, \\ Chennai \\ ${ }^{2}$ Dept. of Electronics and Control Engineering, Sathyabama University, India \\ ${ }^{3}$ Dept. of Information Technology, Government College of Engineering, Karad \\ ${ }^{4}$ Dr. D.Y. Patil School of Engineering, Pune
}

\begin{abstract}
In this research work, the egg laying radius of cuckoo search algorithm is used to create a cluster and then search for the optimum node based on multiobjective genetic algorithm with pareto ranking, so that the data can be forwarded to the sink. The primary focus is onthe two performance metrics parameters, one is the maximization of network lifetime and other is the minimization of delay. For maximizing the network lifetime parameter, the overlapped target sensing by many sensors is wastage of energy by two or more sensors, where the same task can be done by one sensor. To overcome this problem, the sequence set cover methodology is used.For minimization of delay parameter, the sleep-wake scheduling mechanism will be considered, but substantial delays are introduced as transmitting node needs to wait for its next-hop relay node to wake up. These delays can be taken care by developing any cast based packet forwarding schemes where individual node forwards a packet to the first neighboring node that wakes up among multiple candidate nodes. This any cast forwarding schemes minimizes the expected packet-delivery delays from the sensor nodes to the sink node. The introduced work will perform energy proficient routing with an objective to improve the network life, packet loss ratio and overall network throughput. The proposed algorithm was simulated in MATLAB and compared with LEACH algorithm. The results show that our proposed algorithm issuperiorfor prolonging the network lifetime, minimizing the packet loss and increasing the throughput.
\end{abstract}

\section{KEYWORDS:}

Wireless Sensor Network, Energy Efficient Routing, Cuckoo Search, Pareto ranking, Multi-objective Genetic Algorithm,any cast, sleep-wake scheduling.

\section{INTRODUCTION}

Wireless Sensor Networks (WSNs) consist of hundreds of thousands of micro sensorsnodes, which are connected by a wireless medium. These sensor nodes are constrained in power, memory and computational capabilities. To extend the network life time, we normally use energy aware approaches such as multihop communication, in- network data processing, data fusion, and sleep\& wake up methods [1].Clustering based routing techniques are mostly used in WSN applications because of their divide and conquer strategy. As per [2-3], the key element to prolong the network lifetime can be done by balancing the dissipated energy among the available nodes. Hence efficient data clustering and routing techniques should be used to prolong the network lifetime [4]. 
International Journal of Advanced Smart Sensor Network Systems (IJASSN), Vol 7, No.2/3, July 2017

The motive behind this research work is to increase the lifespan of the WSNs as sensor nodes are limited in energy resource and there is no option to recharge them on the go.The main objective of this article is to construct a new energy efficient routing protocol based onCuckoo Search (CS) and multiobjective genetic algorithm with pareto-ranking techniques.

\section{Preliminaries}

\subsection{A BRIEF OVERVIEW OF CUCKOO SEARCH}

Cuckoo search is a meta-heuristic optimization method for solving problems to provide optimal solution.Cuckoo search was first introduced by Xin-She Yang and Suash Deb in 2009[1]. The hypothesis of cuckoo search was motivated by the reproducing conduct of types of bird called cuckoo.

The cuckoo birds lay their eggs in the homes of other host birds. At the point when the hostbirds finds the eggs are not their own, it will either discard these outsider eggs or essentially surrender its home and construct another home somewhere else. Cuckoo search admired such reproducing conduct, and in this way can be related for different optimization issues.

The Cuckoo search works on three idealized energetic standards:

1. One egg is laid by a cuckoo at a particular time, which is placed at anarbitrarily selected nest.

2. The finest nest with extraordinarycaliber of eggs gets carried over to the subsequent generation.

3. The quantity of existing host nests are fixed, and the host bird with a probability $P a \in[0,1]$ discovers the egg laid by a cuckoo.

Taking into account the above three guidelines, the likelihood is that the host bird can either discard the egg or surrender the nest and assemble a totally new nest.

\subsection{A Brief Overview of Multi-objective Genetic Algorithm with Pareto RANKING}

The multi-objective optimization is anarea which encompassesadditional objective function to be optimized concurrently. Multi-objectiveoptimization methodhas been applied in various areas wherein optimal decisions are required in the presence of trade-offs between two or more differing objectives [13-14]. A few ideas of multi- objective improvement issues considered are as below

\subsubsection{A Multi-ObJective DeCision Problem:}

Let $s=\left\{s_{1}, \ldots \ldots, s_{x}\right\}$ be thedecision variablevectorof $x$-dimension, in the solution space $S$. A vector $s^{*}$ is prerequisite to decrease a known set of Mobjective functions $z\left(s^{*}\right)=\left\{z_{1}\left(s^{*}\right), \ldots \ldots, z_{k}\left(s^{*}\right)\right\}$. The solution space $S$ is restricted by asequence of constraints, as $f_{i}\left(s^{*}\right)=c_{i}$ for $i=1, \ldots \ldots \ldots n$, and is bound by the decision variables. 


\subsubsection{DOMINANCE:}

let's say vector $a=\left\{a_{1}, a_{2}, a_{3}, \ldots \ldots, a_{m}\right\}$ dominates vector $b=\left\{b_{1}, b_{2}, b_{3}, \ldots \ldots, b_{m}\right\}$ if and only if $a$ is partially less than $b$.

\subsubsection{PARETO Optimality:}

A solution $Z_{u} \in Z$ is assumed to be pareto optimal if and only if there is not at all $Z_{v} \in Z$ for which $v=f\left(Z_{v}\right)=\left\{v_{1}\right.$ to $\left.v_{m}\right\}$ dominates $u=f\left(Z_{u}\right)=\left\{u_{1}\right.$ to $\left.u_{m}\right\}$.

\subsubsection{PAReto Optimal SET AND Front:}

Let the non-dominated set $D$ where $D \subseteq Z$, is defined as $Z_{p}=\{a \in D \mid a$ is non-dominated regarding $Z\}$.

The objective function values in the objective space are $S_{p}=F\left(Z_{p}\right)=\left\{f(a) \mid a \in Z_{p}\right\}$,

Where $Z_{p}=$ Pareto optimal set

$S_{p}=$ cohere pareto optimal front

The finalobjective of a multi-objective optimization algorithm is to categorize solutions in the Pareto optimal set. So, a usefulmethodfor multi-objective optimization is to discover a set of solutions that signify the Pareto optimal set as much as possible.

\section{RELATED WORK}

Historically nature inspired algorithms have been extensively used in computational intelligence. Many researchers are working in this area and have developed different algorithms serving different purposes. One of the most interesting areas where these algorithms can be used widely is clustering in wireless sensor networks [5]. Still limited energy of node is one the main obstacles; therefore research is going towards the nature inspired algorithms which tend to give better solutions as compared to classical algorithms. This research focuses on cuckoo search for the development of clustering algorithms and multiobjective genetic algorithm for optimum node selection.Cuckoo search was first presented by Xin-She Yang et al. in 2009[6]. The theory of cuckoo search was inspired by the species of bird called cuckoo [7].The concept of laying eggs and breeding of cuckoos has triggered the concept of basic algorithms using cuckoo search. Dhivya et al [8] has suggested a clustering algorithm based on cuckoo search. Theauthors of this article claims that theirimplemented techniqueincreasesthe lifetime of the network by aggregating active nodes to about 15 percent.

Bhatti et al.[9], has proposed cuckoo based energy effective routing in WSN to improve the network throughput and network lifetime without increasing the congestion over the network. The authors used fuzzy systems to modify PEGASIS and named it as fuzzybased PEGASIS whereincuckoo search algorithm is then used for optimization process. The simulations were carried out in matlab simulation software. The parameters considered for simulations are 100 sensor nodes deployed in the 100 by 100 meter square field. The sensor nodes were equipped with 45 meter communication range. The results were measured with respect to number of nodes 
International Journal of Advanced Smart Sensor Network Systems (IJASSN), Vol 7, No.2/3, July 2017

alive, average energy of the network and cost incurred per iterations. The author claims that earlier system's lifetime was $76 \%$ and the proposed system has a lifetime of $92 \%$ with an achievement of $16 \%$ of system lifetime. Dhivya et al [10], the cuckoo based particle approach (CBPA) was proposed to increase energy efficiency in WSN and multimodal functioning. Using Cuckoo search, cluster head is selected and clustersare formed among the sensor nodes. They measured performances such as energy minimization, energy conservation of WSN and lifetime maximization.On comparison of this proposed CBPA with the standard LEACH and HEED protocol it was proved that exhibitedsimulation results were comparable largelybecause of prime search process in cluster formation and also distribution of suitabletracks in transmission of the data sensed.M. Aslam etal [2012] [11] have proposed a Centralized EnergyEfficient Clustering (CEEC) routing protocol. The CEEC was designed for three level heterogeneous WSN.CEEC can be applied to the WSNs with multi-levelheterogeneity. In CEEC, entire network area is separatedinto three equivalent sections such that nodes with equalenergy are located in same section. CEEC has improvedthroughput and network lifetime.A.A. khan et. al. [2012][12] have proposed Heterogeneity-aware Hierarchal StableElection Protocol (HSEP) with two protocols. In clusteringprotocols farther the CS is from BS more is the amount ofenergy consumed during transmission. Proposed protocolis designed to lower the amount of energy consumedduring transmission from $\mathrm{CH}$ toBS. Simulation provedthat the proposed protocol increases network lifetime andstability period as compared to other protocols.

\section{Proposed Work}

The proposed approach uses the concept of cuckoo search algorithm to create a cluster of a WSN. The cluster formation is based on the cuckoo's Egg Laying Radius (ELR). Each cluster consists of two types of nodes namely a trigger node and optimum nodes, where the trigger nodes are responsible to form a cluster. Usually the triggered node is selected based on the highest residual energy and which is relatively close to the event. These trigger nodes are also termed as Cluster Head $(\mathrm{CH})$. Once the cluster is formed around the trigger node considering the ELR. Whenever any event occurs, the data forwarding is taken care by the optimum nodes.Selection of optimal node is based on multiobjective genetic algorithm with pereto ranking concept. The flowchart of the proposed work is presentding figure 1 .

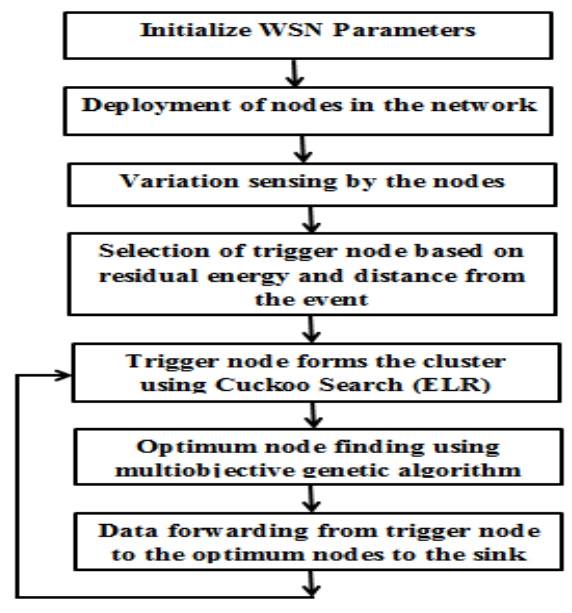

Figure 1: Flow chart of the proposed system 
International Journal of Advanced Smart Sensor Network Systems (IJASSN), Vol 7, No.2/3, July 2017

\subsection{Cluster Formation Using Cuckoos Egg Laying Radius (ELR)}

One of the natural tendenciesof cuckoosis to lay eggs within a maximum distance away from their habitat. This distance from the habitat to the host bird'snest istermed as "Egg Laying Radius (ELR)" as shown in figure2and cuckoo normally laid their eggs within this ELR. When an event occurs (say earthquake, fire, etc.) a node which is having highest residual energy and which is near to the event is selected as a trigger node. Considering this trigger node as the center of the cluster and ELR as the radius, a cluster is formed.

To explain an optimization problem, the problem variables should be in the form of a collection, which is termed as "habitat" in Cuckoo Optimization Algorithm. In a $N_{\text {var }}$-dimensional optimization problem, the habitat is represented as a collection of $1 X N_{v a r}$, which is the present living location of the cuckoo. The collection is stated as

$$
\text { Habitat }=\left[x_{1}, x_{2, \ldots \ldots . . .} x_{N_{v a r}}\right]
$$

Where $\left[x_{1}, x_{2, \ldots \ldots . . .,} x_{N_{v a r}}\right]$ are floating point numbers.

Another natural phenomenonof cuckoos is that, each cuckoo lays about five to twenty eggs. This range is termed as the upper limit $\operatorname{Var}_{h i}$ and lower limitVar ${ }_{l o w}$ of the egg dedication to each cuckoo at different iterations. Therefore ELR is calculated as

$$
E L R=\alpha \mathrm{X} \frac{\text { current number of eggs }}{\text { total number of eggs }} \mathrm{X}\left(\text { var }_{\text {hi }}-\text { var }_{\text {low }}\right)
$$

Where $\alpha=$ integer, handles the maximum value of ELR.

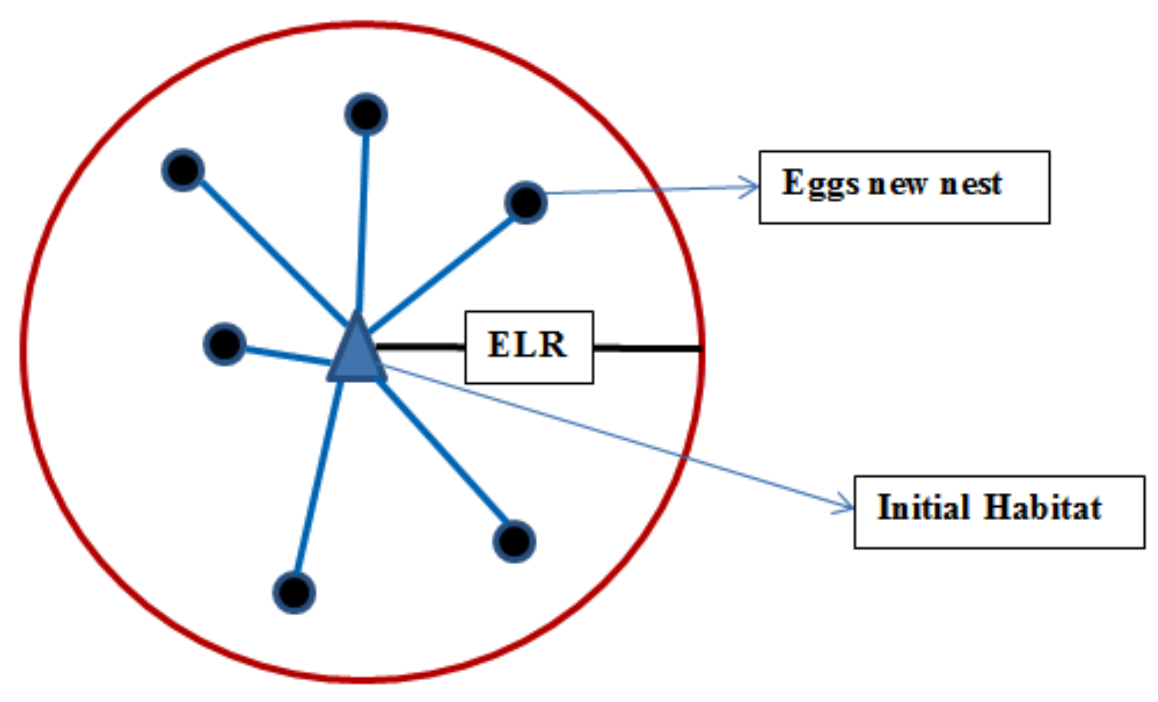

Figure 2: The Egg Laying Radius with six eggs. 


\subsection{Finding Of Optimum Node Using Multi-ObJective Genetic Algorithm}

For the discovery of optimum node, the multi-objective genetic algorithm based on Pareto ranking is taken into consideration. The efficiency of multi-objective genetic algorithm mainly depends on the selection of fitness functions. Here for the selection of fitness functions say $F_{1}$ and $\mathrm{F}_{2}$ are considered, where $F_{1}$ takes care for maximization problems and $F_{2}$ takes care for minimization problems. The same can be compared with WSN, where we need to maximize $\left(F_{1}\right)$ the lifetime of the network and minimize $\left(F_{2}\right)$ the delay in data transmission.In this we need to take care of more than one objective say $\left(F_{1}\right)$ and $\left(F_{2}\right)$, so we need to use multiobjective genetic algorithm to find the optimum node, as the multiobjective genetic algorithm gives very good performance to solve the maximization and minimization problems.

Following analysis are carried out on $\left(F_{1}\right)$ in 4.2 .1 and $\left(F_{2}\right)$ in 4.2 .2 for fitness so that $\left(F_{1}\right)$ takes care for maximization problem and $\left(F_{2}\right)$ for minimization problem.

\subsubsection{SElECTION OF $\left(F_{1}\right)$ FOR MAXIMIZATION}

The objective here is that, we need to set the parameters of $\left(F_{1}\right)$ in such a way that the output of $\left(F_{1}\right)$ is maximized. There we need to prove that

$$
F_{1}=\text { Maximize }\left(C_{1}+C_{2}+\cdots C_{n}\right)
$$

Where $C_{n}=$ Sequence Set Cover (SSC).

The SSC is represented as $C_{i}=\left\{\left(N_{1}, r_{1}, e_{1}\right), \ldots \ldots \ldots\left(N_{i}, r_{i}, e_{i}\right)\right\}$, where $i<\infty, N_{i}$ is using sensing range $r_{i}$ to sense the target $t_{i}$ with energy $e_{i}$.

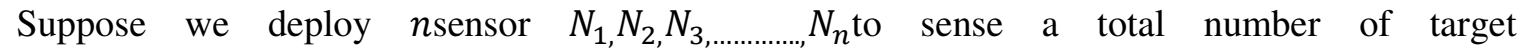
mas $\left(t_{1}, t_{2, \ldots \ldots . . .} t_{m}\right)$,where each node has multiple sensing range $r_{1}, r_{2}, r_{3, \ldots \ldots \ldots . . .} r_{p}$ and energy consumption $e_{1}, e_{2}, e_{3, \ldots \ldots . . . . .} e_{p}$ for each node for each sensing range. Assuming that the maximum energy associated with each node is $E$.

Our main objective was to find the efficient sequence of node and sensing range so that utilization of power is maximized and at the same time all targets should be covered.

For example let's consider three sensor nodes $N_{1}, N_{2}$ and $N_{3}$ as shown in the figure 3.Node $N_{1}, N_{2}$ and $N_{3}$ has sensing range $r_{1} r_{2}$ and $r_{3}$ respectively which are overlapped with each other. As we can see from figure 3 , there are two targets $t_{1}$ and $t_{2}$ which come under the range of more than one sensor. Suppose $t_{1}$ is the first target to sense which comes under the range of $N_{1}$ and $N_{2}$ sensors. In this case for sensing the same target by $N_{1}$ and $N_{2}$ both will utilize their energy which is waste of energy and will lead to reduce the network lifetime.To overcome this problem we assumed the following techniques:

First we will try to identify dissimilar sequence of nodes and sensing ranges to cover the set of targets. Therefore $C_{i}=\left\{\left(N_{1}, r_{1}, e_{1}\right), \ldots \ldots \ldots\left(N_{i}, r_{i}, e_{i}\right)\right\}$, where $i<\infty, N_{i}$ is using sensing range $r_{i}$ to sense the target $t_{i}$ with energy $e_{i}$. 
Continue with figure 3, we observe that target $t_{1}$ is in the range of $N_{1}$ and $N_{2}$ and target $t_{2}$ is in the range of $N_{2}$ and $N_{3}$. In this scenario we need to calculate two Sequence Set Cover as $C_{1}$ and $C_{2}$. Therefore $C_{1}=\left\{\left(N_{1}, r_{i}, e_{i}\right),\left(N_{2}, r_{j}, e_{j}\right)\right\}$ and $C_{2}=\left\{\left(N_{2}, r_{j}, e_{j}\right),\left(N_{3}, r_{k}, e_{k}\right)\right\}$.Theresidual energy of $N_{1}, N_{2}$ and $N_{3}$ are $\left(E-e_{i}\right),\left(E-e_{j}\right)$ and $\left(E-e_{k}\right)$ respectively.

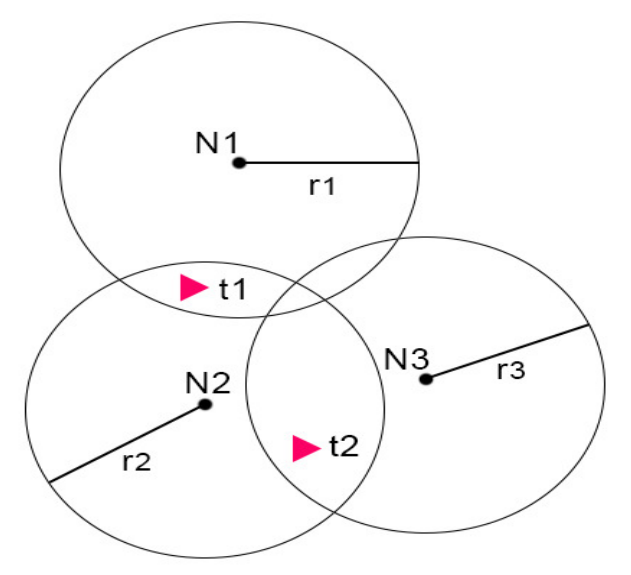

Figure3: Nodes deployment and sensing range

Now suppose we assume each Sequence Set Cover as a single round in WSN, then the network lifetime will be maximum if and if only when the network can sustain for the more number of rounds. In other word we can say that lifetime of network will be more if $C_{1}+C_{2}+\cdots C_{n}$ is maximum.So, our first objective function

\section{Subject to:}

$$
F_{1}=\operatorname{Maximize}\left(C_{1}+C_{2}+\cdots C_{n}\right)
$$

i. $\quad$ Energyspent by every sensor node is $\leq$ total initial battery energyof the sensor nodes.

ii. If the cover $k$ consists of a sensor node ${ }_{i}$, then exactly one of its $P$ sensing ranges are positioned.

iii. This assures that each of the targetstjgets covered by each set $c k$.

\subsubsection{SELECTION OF $\left(F_{2}\right)$ FOR MinimizATION}

The issue of delay minimization is an instance of the stochastic shortestroute issue, wherein thenode, which holds the packet, assesses the present state.The resultant disturbance relates to the costwhich needs to be minimized.In the event that there is just a single source creating the eventreportingpackets, the end-to-end disturbance of the first packet is figured as an element of any cast strategy $(X, Y)$ and furthermore, the rest the sleep-wake schedules policy $s p$. The any caststrategy $(X, Y)$ is defined by forwarding candidate node sets $(X)$ and priority of node $(Y)$. The responsibility of the forwarding node set, which is a set of candidates nodes is to forward a packet at node. Matrix $X$ contains all the forwarding set of entire nodes denoted by

$$
X=\left[x_{k l}, k=1, \ldots . . M, l=1, \ldots . . M\right]
$$

Where $x_{k l}=1$ if $l$ is in node $k^{\prime}$ sforwarding set

$$
x_{k l}=0 \text { otherwise }
$$


International Journal of Advanced Smart Sensor Network Systems (IJASSN), Vol 7, No.2/3, July 2017

The priority of matrix of nodes are represented as

$$
Y=\left[y_{k l}, k=1, \ldots . . M, l=1, \ldots . M\right]
$$

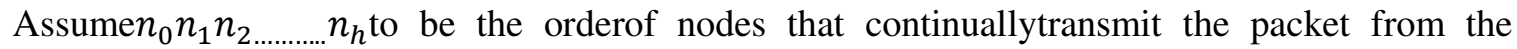
sourcenode $n_{0}$ to base stations.

The order is irregular on the grounds that at each hop, the primary node in the forwarding set that awakens is chosen as a next-hop node.

If thepacket arrives atsink $s$ after $H$ hops, then we haven $n_{h}=s$ forh $\geq H$.Let $d_{i}(s p, X, Y)$, is the expected one-hop delayat node $i$ under the anycaststrategy $(X, Y)$, i.e. theexpected delay from the time the packet arrives atnode $i$ to the time it is forwarded to the next-hop node.

Then, the end-to-end delay for $\operatorname{packet} D_{i}(s p, X, Y)$ from node $i$ to sink $s$ isexpressed as

$$
D_{i}=(s p, X, Y)=E\left[\sum_{k=0}^{\infty} d_{i k}(s p, X, Y)\right]
$$

So, our second objective function $F_{2}=\operatorname{Minimize} D_{i}=\operatorname{Minimize}\left(E\left[\sum_{k=0}^{\infty} d_{i k}(s p, X, Y)\right]\right)$. After finding both the objective function $F_{1}$ and $F_{2}$, now we need to discover the pareto ranking. In this calculation we try to locate the rank of the points, which is equivalent to the quantity of points by which it is dominated in the present population.Pareto ranking algorithm is demonstrated in algorithm 1 .

Algorithm 1:Pareto Ranking Algorithm

$\mathbf{P R}\left(\mathrm{p}_{\mathrm{tp}}, \mathrm{d}_{\mathrm{n}}\right) \quad / / \mathrm{p}_{\mathrm{tp}}$ total number of points in the population, $\mathrm{d}_{\mathrm{n}}$ number ofdimensions

1. $\left\{\mathrm{T}_{1}\left[\mathrm{p}_{\mathrm{tp}}\right]=0, \mathrm{~T}_{2}=0, \mathrm{~T}_{3}=0\right.$. // Initialization of variables $\&$ array

2. $\mathrm{A}_{1}\left[\mathrm{p}_{\mathrm{tp}}\right]\left[\mathrm{d}_{\mathrm{n}}\right]=$ Population

3. for $\left(\mathrm{i}=0\right.$ to $\left.\left(\mathrm{p}_{\mathrm{tp}}-1\right)\right)$

4. $\left\{\right.$ for $\left(\mathrm{j}=0\right.$ to $\left.\left(\mathrm{p}_{\mathrm{tp}}-1\right)\right)$

5. $\{$ if $(\mathrm{j} !=\mathrm{i})$

6. for $\left(\mathrm{k}=0\right.$ to $\left.\left(\mathrm{p}_{\mathrm{tp}}-1\right)\right)$

7. $\left\{\right.$ if $\left(\mathrm{f}_{1}\left(\mathrm{~A}_{1}[\mathrm{j}, \mathrm{k}]\right) \leq \mathrm{f}_{2}\left(\mathrm{~A}_{1}[\mathrm{i}, \mathrm{k}]\right)\right)$

8. $\quad$ if $\left(\mathrm{f}_{1}\left(\mathrm{~A}_{1}[\mathrm{j}, \mathrm{k}]\right)<\mathrm{f}_{2}\left(\mathrm{~A}_{1}[\mathrm{i}, \mathrm{k}]\right)\right)$

9. $\left\{\mathrm{T}_{2}=\mathrm{T}_{2}+1\right\}$

10. else

11. $\left.\left\{\mathrm{T}_{3}=\mathrm{T}_{3}+1\right\}\right\}$

12. if $\left(\left(\mathrm{T}_{2}+\mathrm{T}_{3}\right)==\mathrm{d}_{\mathrm{n}}\right)$

13. $\left\{\mathrm{T}_{1}[\mathrm{i}]=\mathrm{T}_{1}[\mathrm{i}++]\right.$

14. $\left.\mathrm{T}_{2}=\mathrm{T}_{3}=0\right\}$

15. $\}\}\}$ 
International Journal of Advanced Smart Sensor Network Systems (IJASSN), Vol 7, No.2/3, July 2017

Once the front is discovered, we can say that the optimal node will be a member of the front and then we can apply genetic algorithm to this front i.e. encoding, cross over, mutation. The adaptive strategy algorithm is demonstrated in algorithm 2.

Algorithm 2: Adaptive Strategy Algorithm

1. $\left\{\mathrm{N}_{\mathrm{b}}[\mathrm{N}]=0 / / \mathrm{N}_{\mathrm{b}}\right.$ : is an array //

2. Int $\mathrm{s}_{1}=0$,int $\mathrm{s}_{2}=0$;

3. Float $\mathrm{z}=0.0, \mathrm{q}=0.0$;

4. Enter number of nodes $\mathrm{N}$

5. For $(\mathrm{i}=0$ to $\mathrm{N}-1)$

6. $\left\{\right.$ Enter $\mathrm{N}_{\mathrm{b}}[\mathrm{i}] / / / \mathrm{N}_{\mathrm{b}}[\mathrm{i}]$ is the no of mutation done by an $\mathrm{i}^{\text {th }}$ mutation operator in a generation //

7. For $\left(\mathrm{j}=0\right.$ to $\left.\left(\mathrm{N}_{\mathrm{b}}[\mathrm{i}]-1\right)\right)$

8. $\{\mathrm{p}[\mathrm{i}, \mathrm{j}]=\mathrm{Mx}[\mathrm{f}(\operatorname{rank}(\mathrm{j})), \mathrm{f}(\operatorname{mut}(\operatorname{Rk}(\mathrm{j})))]-\mathrm{f}(\operatorname{Rk}(\mathrm{j}))$;

9. $\}\}$

10. for $(\mathrm{i}=0$ to $(\mathrm{N}-1))$

11. $\left\{\right.$ for $\left(\mathrm{k}=0\right.$ to $\left.\left(\mathrm{N}_{\mathrm{b}}[\mathrm{i}]-1\right)\right)$

12. $\left\{\right.$ for $\left(\mathrm{j}=0\right.$ to $\left.\left(\mathrm{N}_{\mathrm{b}}[\mathrm{i}]-1\right)\right)$

13. $\left\{\mathrm{s}_{1}=\mathrm{s}_{1}+\mathrm{p}[\mathrm{k}, \mathrm{j}] ;\right\}$

14. $\mathrm{z}=\left(\mathrm{s}_{1} / \mathrm{N}_{\mathrm{b}}[\mathrm{k}]\right)+\mathrm{Z}$;

15. $\mathrm{s}_{2}=\mathrm{s}_{2}+\mathrm{p}[\mathrm{i}, \mathrm{k}]$;

16. $\mathrm{s}_{1}=0$;

17. $\}$

18. $\mathrm{q}=\left(\mathrm{s}_{2} / \mathrm{N}_{\mathrm{b}}[\mathrm{i}]\right)$;

19. $\mathrm{t}[\mathrm{i}]=\mathrm{q} / \mathrm{z}$;

20. $\}$

21. for $(\mathrm{i}=0$ to $(\mathrm{N}-1))$

22. $\left\{\mathrm{R}[\mathrm{i}]=\mathrm{t}[\mathrm{i}] *\left(\mathrm{P}_{\mathrm{mut}}-\mathrm{N}\right)\right.$

23. \}\}

/**** Function for maximum ****/

1. $M x($ inta,int $b)$

2. \{

3. If $(a>=b)$

4. Return a

5. Else

6. return $b$

7. $\}$

/**** Function for ranking $* * * * /$

1. $\mathrm{Rk}$ (int $\mathrm{y})$

2. \{

3. Intz $[n][2]=0$, temp $=0, c=0$;

4. $\operatorname{For}(\mathrm{i}=0$ to $(\mathrm{n}-1))$

5. \{ 
International Journal of Advanced Smart Sensor Network Systems (IJASSN), Vol 7, No.2/3, July 2017

6. $\operatorname{Ar}[\mathrm{i}][0]=\mathrm{r}[\mathrm{i}]$

7. $\operatorname{Ar}[\mathrm{i}][1]=\mathrm{i}$;

8. $\}$

9. $\operatorname{For}(\mathrm{i}=0$ to $(\mathrm{n}-2))$

10. \{

11. $\operatorname{For}(\mathrm{j}=(\mathrm{i}+1)$ to $n-1)$

12. $\{\operatorname{If}(z[i][0]<z[j][0])$

13. \{

14. $\mathrm{Temp}=\mathrm{z}[\mathrm{i}][0]$;

15. $\operatorname{Ar}[\mathrm{i}][0]=z[\mathrm{j}][0]$;

16. $\operatorname{Ar}[\mathrm{j}][0]=\mathrm{Tp}$

17. $\mathrm{Tp}=\mathrm{z}[\mathrm{i}][1]$;

18. $\mathrm{z}[\mathrm{i}][1]=\mathrm{z}[\mathrm{j}][1]$;

19. $z[j][1]=T p$;

20. $\}\}\}$

21. $\mathrm{C}=\mathrm{z}[\mathrm{y}][1]$;

22. Return $(\mathrm{a}[\mathrm{c}])$;

23. \}

After some iteration, we will notice that the nodes of the front have become fixed. Now we can choose any member of node as an optimal node. Now once this is done the data can be sent through the optimal to the sink.

\section{Performance Analysis Of Proposed System}

The execution of the proposed algorithm has been simulatedin Matlab. Table 1 lists the simulation parametersto create a simulation environment.

\subsection{Simulation Parameters}

\begin{tabular}{|l|l|}
\hline \multicolumn{1}{|c|}{ General Parameters } & \multicolumn{1}{c|}{ Values } \\
\hline Area & $500 \mathrm{X} \mathrm{500 \textrm {M } ^ { 2 }}$ \\
\hline No. of nodes & 500 \\
\hline Initial Energy & 100 Joules \\
\hline Data Packet Size & 40 bytes \\
\hline Sleep Power & $0.0006 \mathrm{~mW}$ \\
\hline No. of rounds & 100 \\
\hline No. of Nest & 100 \\
\hline No. of eggs in the nest & $1-5$ \\
\hline
\end{tabular}

\begin{tabular}{|l|l|}
\hline \multicolumn{1}{|c|}{ GA Parameters } & \multicolumn{1}{c|}{ Values } \\
\hline Max. Generation & 500 \\
\hline Population size & 200 \\
\hline Length of chromosomes & 100 \\
\hline Mutation rate & $0.007 / 0.1$ \\
\hline Crossover rate & 0.7 \\
\hline
\end{tabular}

Table 1: Simulation Parameters 


\subsection{RESUlTS AND Discussions}

The hybrid model consisting of CS and Multiobjectivegenetic algorithms yields better results as compared to LEACH in terms of better execution ofnetwork lifetime, packet loss ratio and throughput.

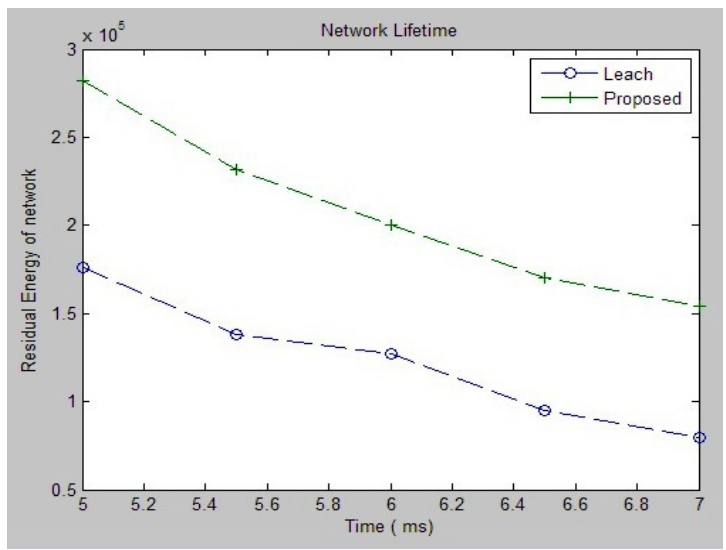

Figure 4. Network Lifetime

Figure 4 shows the graph of residual energy of network with respect to the time in milliseconds (Network lifetime) for proposed algorithm and LEACH. From Figure 4 it is evident that our proposed algorithm has the capability to keep alive the network for an extended period as compared to LEACH.

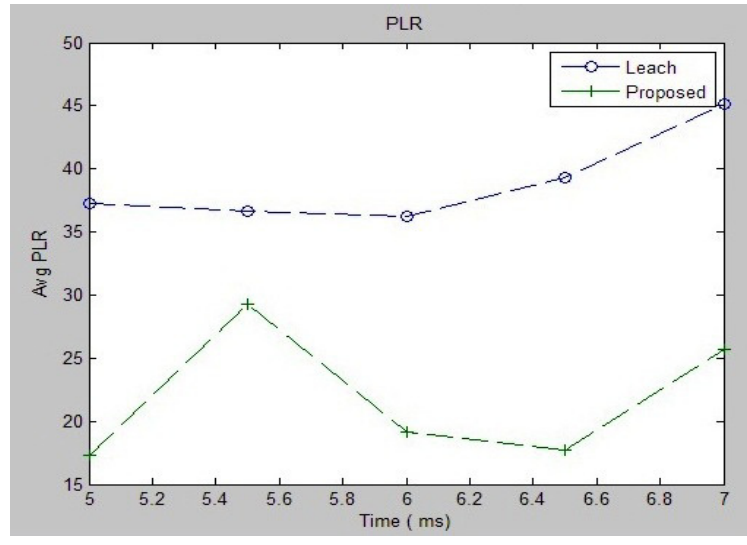

Figure 5.Packet Loss Ratio

Figure5 shows the average Packet Loss Ratio (PLR) with respect to the time in milliseconds for the proposed algorithm and LEACH. Figure 5 shows that the proposed algorithm is better in terms of average PLR (which is less than LEACH). 
International Journal of Advanced Smart Sensor Network Systems (IJASSN), Vol 7, No.2/3, July 2017

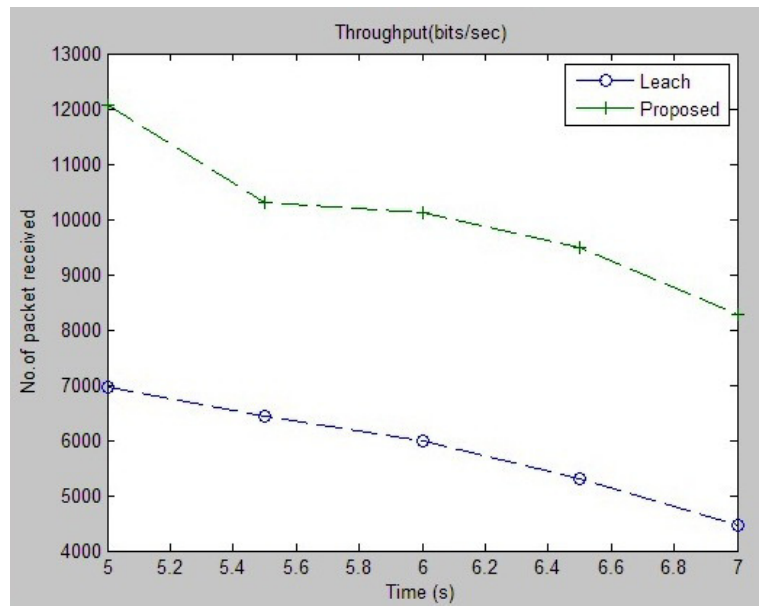

Figure 6. Throughput

Figure 6 illustrates the gained efficiency in terms of throughputof the network. From Figure6, it is evident that our proposed algorithm is superior toLEACH in terms of improved throughput.

\section{Conclusion}

In this paper, a novel opportunistic routing protocol has been presented, which introduces hybrid method based on cuckoo search and multiobjective genetic algorithm with pareto ranking for clustering and routing. The proposed algorithmis energy efficient as it considers the effect of each transmission and reception of packets on node's total energy. The simulation and performance analysis has been done by comparing proposed algorithmwith LEACH algorithm. The results show that proposed algorithm has good performance in the presence of nodes. The proposed method optimizes the network lifetime, packet loss ratio and throughput in WSN. In the future we can consider more parameters and metrics components to improve network performance by considering other properties of the WSN, cuckoo search and multiobjective genetic algorithm. The result also proves that the proposed method is better than LEACH algorithm and increases the lifetime of the network.

\section{REFERENCES}

[1] Xin-She Yang and Suash Deb, "Cuckoo search via Levy flights", World Congress in nature and biologically inspired computing, 2009, NaBIC 2009. Pp 210-214, IEEE, 2009.

[2] Glue D, and Morgan R, (1972), “Cuckoo host in british habitats, bird study, 19(4), 187-192.

[3] Akyildiz I.F, Su W. Sankarasubramaniam, Y Cayira. E "Wireless sensor network: A survey", Computer networks 38, 393-422(2002).

[4] Akojwar A.G and Patrikar R.M. "Improving life time of wireless sensor networks using natural network based classification techniques with cooperative routing", international journal of communication, 2(1), 75-86(2008). 
International Journal of Advanced Smart Sensor Network Systems (IJASSN), Vol 7, No.2/3, July 2017

[5] Raghuchandra R. Goudar et al. , "Clustering approach for Wireless Sensor Networks based on Cuckoo Search Strategy", International Journal of Advanced Research in Computer and Communication EngineeringVol. 3, Issue 6, June 2014.

[6] Chakraborty W, Chakraborty A, Mitra S.K and Naskar M.K, “ An energy efficient scheme for data gathering in WSNs using particle swarm optimization, Journal of applied computer science 6(3), 9-13(2009).

[7] R. Rajabioun, "Cuckoo Optimization Algorithm," Applied Soft Computing. vol. 11, Issue 8, 2011, pp. 5508-5518.

[8] Dhivya, M., Sundarambal, M., \& Vincent, J. O. (2011). Energy Efficient Cluster Formation in Wireless Sensor Networks Using Cuckoo Search.Swarm, Evolutionary, and Memetic Computing Lecture Notes in Computer Science, 140-147. doi:10.1007/978-3-642-27242-4_17

[9] Gurpreet Kaur Bhatti et al. ," Cuckoo based Energy Effective Routing in Wireless Sensor Network", International Journal of Computer Science and Communication Engineering Volume 3 Issue1 (February 2014 issue).

[10] Dhivya, M., Sundarambal, M., \& Anand, L.N., "Energy Efficient Computation of Data Fusion in Wireless Sensor Networks using Cuckoo based particle approach(CBPA)", IJCNS international journal of communications, Network and system sciences, 04(04), 2011, pp 249-255. Doi:10.4236/ijcns.2011.44030

[11] Aslam, M., T. Shah, Nadeem Javaid, A. Rahim, Z. Rahman, and Z. A. Khan. "CEEC: Centralized energy efficient clustering a new routing protocol for WSNs." In Sensor, Mesh and Ad Hoc Communications and Networks (SECON), 2012 9th Annual IEEE Communications Society Conference on, pp

[12] Khan, Adnan Ahmed, Nadeem Javaid, Umar Qasim, Zhi Lu, and Zahoor Ali Khan. "HSEP: heterogeneityaware hierarchical stable election protocol for WSNs." In Broadband, Wireless Computing, Communication and Applications (BWCCA), 2012 Seventh International Conference on, pp. 373-378.IEEE, 2012.

[13] GokceHocioglu, VahidFaryad,AghjehKand, ErhanSesli, "Multiobjective clustering for wireless sensor networks", expert systems with application Elsevier, 2016,59,86-100.

[14] Sanjeev Wagh, Ramjee Prasad, "Heuristic Clustering for Wireless Sensor Networks using Genetic Approach "International Journal of Wireless and Mobile Networking (IJWAMN)", Vol. 1, No. 1(November 2013). 